Supporting Information

\title{
Enhancing Mechanical and Combustion Performance of Boron/Polymer Composites via Boron Particle
}

\section{Functionalization}

Yue Jiang ${ }^{1}$, Nil Ezgi Dincer Yilmaz ${ }^{1}$, Kayla P. Barker ${ }^{2}$, Jihyun Baek ${ }^{1}$, Yan Xia ${ }^{2}$ * and Xiaolin Zheng ${ }^{1, *}$

${ }^{1}$ Department of Mechanical Engineering, Stanford University, Stanford, CA 94305, USA

${ }^{2}$ Department of Chemistry, Stanford University, Stanford, CA 94305, USA 


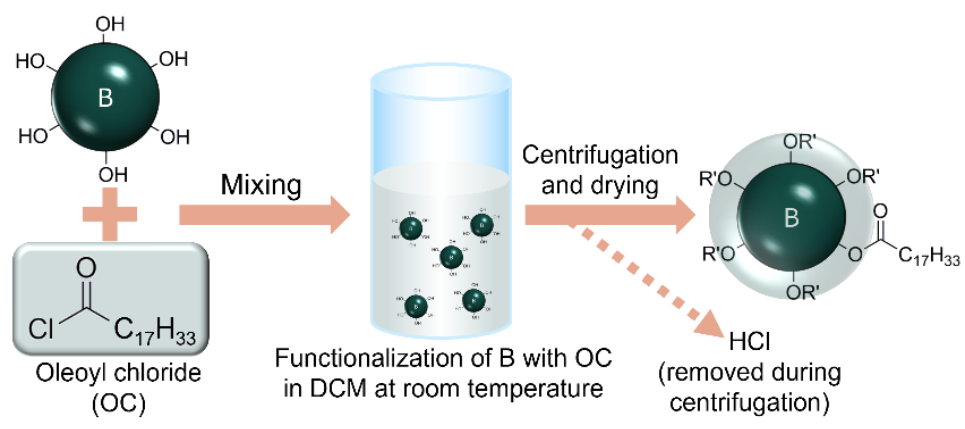

Figure S1. Schematics of the synthesis procedure for OC-B particles. Briefly, B particles were sonicated and dispersed in DCM solvent. OC was then added to the solution and the mixture was further sonicated. The $\mathrm{B} / \mathrm{DCM} / \mathrm{OC}$ suspension was vigorously stirred via a magnetic bar, forming OC functionalized B particles (OC-B). The OC-B particles were washed several times with DCM to remove excess $\mathrm{OC}$ and $\mathrm{HCl}$, and then centrifuged and further dried overnight to remove DCM. 

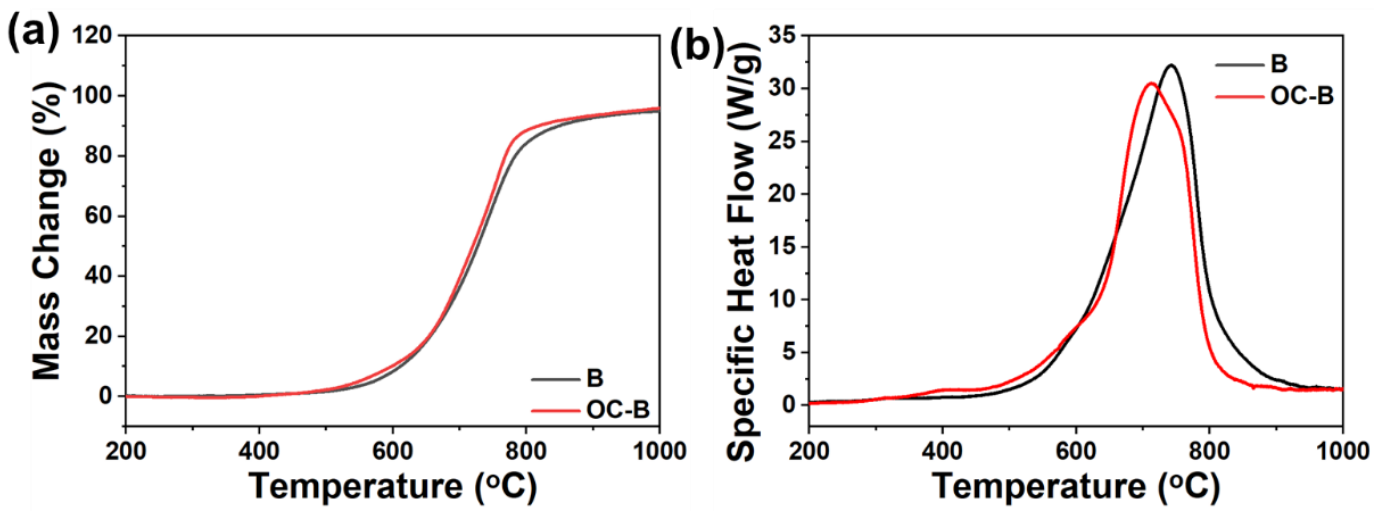

Figure S2. Thermochemical properties of pristine B and OC-B particles investigated by (a) TGA and (b) DSC. Generally, pristine B and OC-B exhibit similar mass change, heat flow profile, and integrated heat release values $(30.5 \mathrm{~kJ} / \mathrm{g}$ and $32.3 \mathrm{~kJ} / \mathrm{g})$. Conditions: $10 \mathrm{mg}$ of sample, heating rate of $10^{\circ} \mathrm{C} / \mathrm{min}$, air flow (40 sccm). 


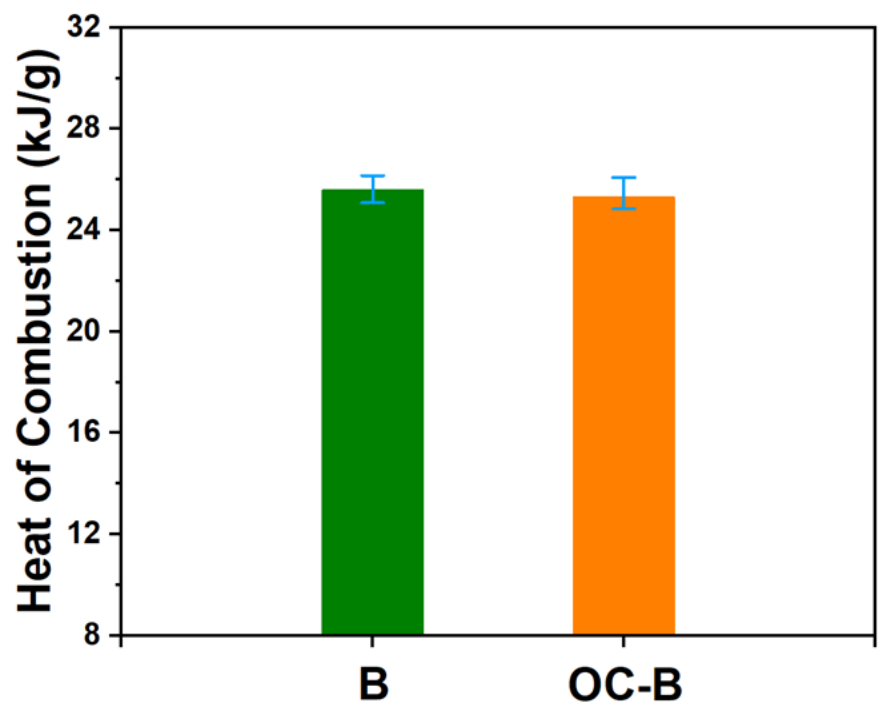

Figure S3. The heat of combustion of pristine B and OC-B particles acquired by bomb calorimeter. The measured heat of combustion of OC-B is very close to that of pristine B particles, suggesting that the covalently bonded OC layer has little effect on the heat of combustion of B particles. The error bars indicate the variation from three different measurements. Conditions: $20 \mathrm{~atm} \mathrm{O}_{2}$ and 50 mg of powder. 